\title{
Effect of Systemic Doxycycline Administration on the Subgingival Microbial Flora: A Dark-field Microscopy Study
}

\author{
Nermin YAMALIK ${ }^{1}$, Ferda TUNÇKANAT ${ }^{2}$, \\ Tamer ATAOḠLU ${ }^{3}$ and Dilek SENGÜN ${ }^{1}$
}

(Received 11 January and accepted 12 February 1991)

Key words: doxycycline, subgingival flora, dark-field microscopy

\begin{abstract}
The effect of systemically administered doxycycline hydrochloride on subgingival microbial plaque morphotypes was evaluated using dark-field microscopy. After 1 week of drug administration, while the percentage of coccoid bacteria showed a significant increase, the percentages of spirochetes, motile rods and non-motile rods tended to decrease. Since spirochetes and/or motile rods are considered to be associated with the pathogenesis of periodontal disease, the significant decrease in the proportions of these bacteria are conisidered to be important. Therefore, it is suggested that doxycycline may be beneficial for the treatment of periodontal disease as an adjunct to traditional therapeutic methods.
\end{abstract}

\section{Introduction}

It has been well demonstrated that microorganisms play an important role in the pathogenesis of periodontal disease ${ }^{[1-5]}$.

Although the microorganisms responsible have not yet been definitely identified, spirochetes and motile rods are suggested to be associated with periodontal disease $\mathrm{e}^{[1,2,6]}$. There is evidence that an increase in the severity of gingivitis is accompanied by an increase in the percentage of spirochetes and motile rods in the bacterial plaque ${ }^{[2,7,8]}$. LISTGARTEN and LEVIN ${ }^{[2]}$ reported that pockets with a high percentage of spirochetes and motile rods had a greater chance of further periodontal breakdown than pockets with a low percentage of these bacteria.

Systemically administered antibacterial agents are being tried with increasing frequency for the clinical management of periodontal problems ${ }^{[1,7,9-12]}$. Tetracyclines are one group of antibiotics that have been used widely in the treatment of periodontal disease ${ }^{[7,9,11,12]}$.

Doxycycline is a semisynthetic antibiotic derived from oxytetracycline, which can penetrate most body tissues and fluids better than other currently available tetracyclines ${ }^{[13]}$. Doxycycline is primarily bacteriostatic in its action, and exerts its

1 Department of Periodontology, Faculty of Dentistry, University of Hacettepe-Ankara/TURKEY.

2 Department of Microbiology, Faculty of Medicine, University of Hacettepe-Ankara/TURKEY.

3 Department of Periodontology, Faculty of Dentistry, University of Selçuk-Konya/TURKEY.

To whom all correspondence should be addressed: Nermin YAMALIK, Department of Periodontology, Faculty of Dentistry, University of Hacettepe, Ankara, TURKEY. 
antimicrobial effect by inhibition of protein synthesis. It is active against a wide range of gram-positive and gram-negative organisms ${ }^{[14]}$.

The aim of the present study was to investigate the effect of systemically administered doxycycline hydrochloride on microorganisms present in subgingival microbial plaque from patients with periodontal problems, and on the clinical status of periodontal disease.

\section{Materials and Methods}

Selection of patients and experimental oral area: The study was conducted on 28 teeth from 7 patients with a mean age of $33.7 \pm 6.9 \mathrm{yr}$, exhibiting extensive periodontal disease. Care was taken to ensure that none of the patients had received any periodontal treatment and/or antibiotics in the previous 6 months. Patients with systemic disorders were excluded, and all of the patients were informed about the experimental design. The most badly affected teeth in these patients were chosen for the study.

Clinical studies: Following the elimination of supragingival plaque from the experimental tooth surfaces, subgingival microbial plaque samples were obtained using sterile curettes, according to the method of Listgarten and HeLLdeN ${ }^{[16]}$. Each plaque sample was immediately transferred to a sterile glass bottle containing saline. After the subgingival microbial plaque sampling procedures, gingival index (GI) and plaque index (PI) scores of LöE and SILLNESS ${ }^{[15]}$ were recorded for each patient. Furthermore, pocket depth (PD) and presence of bleeding on provocation were determined in the experimental area.

Laboratory studies: Using a sterile syringe, the plaque sample in the bottle was homogenized and a drop of the suspension was placed on a microscope slide and covered with a coverslip. Microorganisms in the samples were counted using a modification of the method of Listagarten and HelldeN ${ }^{[16]}$. Microorganisms including coccoid bacteria, motile rods, spirochetes and other non-motile rods were counted by dark-field microscopy at x600 magnification. The number of microorganisms was calculated and expressed as a percentage.

Drug administration: The patients were recommended to use doxycycline 100 mg twice a day for a period of 7 days. In order to examine solely the antimicrobial effect of the drug, no therapy other than the antibiotic was recommended, including oral hygiene instructions and/or periodontal treatment. After 7 days, subgingival plaque sampling was performed on the same experimental tooth surface once again, and the laboratory procedures were repeated.

Statistical studies: The differences in the clinical parameters and bacterial proportions between the pre-treatment and post-treatment phases were evaluated using Wilcoxon's Signed Rank Test ${ }^{[17]}$.

\section{Results}

Clinical findings: The mean GI and PI scores were $2.35 \pm 0.47$ and $1.82 \pm 0.75$ in the pre-treatment phase. Mean PD was $6.57 \pm 1.39 \mathrm{~mm}$ and bleeding was present in all of the experimental areas. The mean GI and PI scores following drug administration were $2.25 \pm 0.41$ and $1.75 \pm 0.51$, and the mean PD was $6.48 \pm 1.31 \mathrm{~mm}$. 
Although a slight decrease in GI, PI and PD scores was noticed following doxycycline administration, this was not statistically significant $(P>0.05)$, and bleeding was still present in the post-treatment phase.

Microbiological findings: Data on the percentages of bacterial forms before and after doxycycline administration are presented in Table 1 . The mean percentage of coccoid bacteria in the pre-treatment phase was $67.42 \pm 3.19 \%$. Following drug administration, this percentage reached $92.20 \pm 1.30 \%$, the increase being statistically significant $(\mathrm{p}<0.05)$. The mean percentages of spirochetes before and after treatment were $17.62 \pm 2.40 \%$ and $0.39 \pm 0.22 \%$, respectively. The mean percentages of motile rods and non-motile rods in the pre-treatment phase were 8 . $45 \pm 1.27 \%$ and $6.50 \pm 1.15 \%$ (Fig. 1). In the post-treatment phase, the corresponding percentages were $5.09 \pm 0.98 \%$ and $1.95 \pm 0.42 \%$, respectively. The decreases noticed in the percentages of spirochetes, motile rods and non-motile rods during

Table 1: Percentages of bacteria in subgingival microbial plaque samples before and after doxycycline hydrochloride administration

\begin{tabular}{lccccc}
\hline & \multicolumn{2}{c}{ Pre-treatment } & Phase & & \multicolumn{2}{c}{ Post-treatment Phase } \\
\cline { 2 - 3 } \cline { 5 - 6 } & Mean & s.d. & & Mean & s.d. \\
\hline Coccoid Bacteria & 67.42 & 16.92 & & 92.20 & 6.88 \\
Spirochetes & 17.62 & 12.74 & & 0.39 & 1.17 \\
Motile rods & 8.45 & 6.75 & & 5.09 & 5.20 \\
Non-motile rods & 6.50 & 6.11 & & 1.95 & 2.26 \\
\hline
\end{tabular}

s.d. : standard deviation

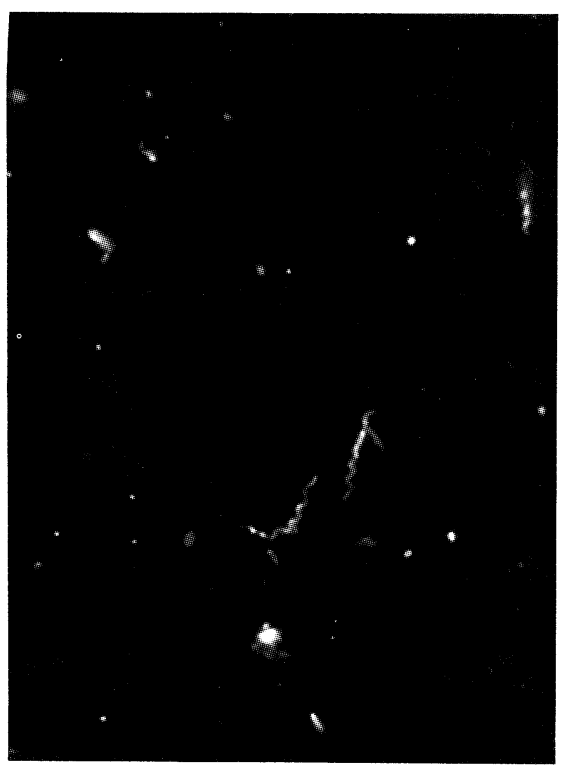

Fig. 1 Subgingival microbial plaque sample in the pre-treatment phase. $(\times 1000)$ 


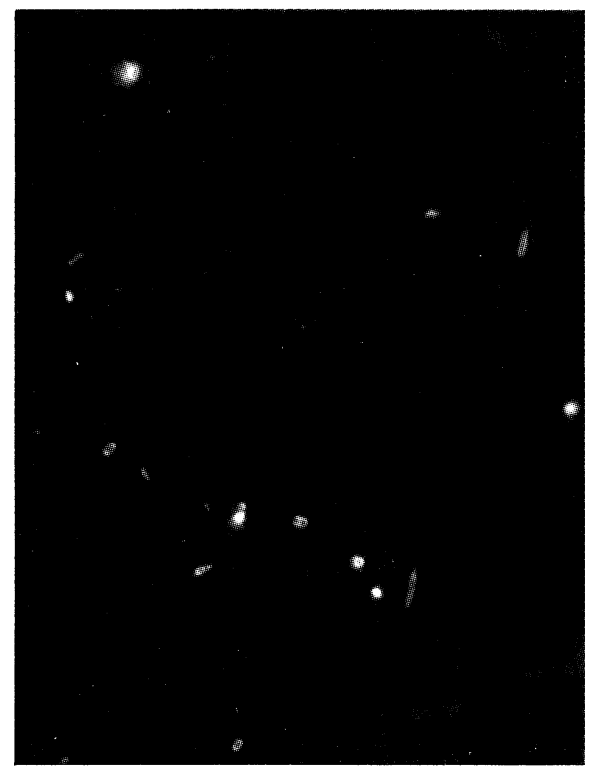

Fig. 2 Subgingival microbial plaque sample following systemic doxycycline administration. $(\times 1000)$

the post-treatment phase were also statistically significant $(\mathrm{p}<0.05)($ Fig. 2).

\section{Discussion}

It has been shown that the microbial flora in healthy and periodontally diseased sites differs significantly. Spirochetes and motile rods are suggested to be related to the severity of periodontal disease ${ }^{[6,8,16]}$. An increased number of spirochetes and gram-negative motile bacteria in diseased sites, and a predominance of gram-positive coccoid bacteria in clinically healthy sites has been demonstrat$\mathrm{ed}^{[6,8,16,18]}$. There is evidence that an increase in the severity of gingivitis is accompanied by an increase in the percentage of spirochetes in the bacterial plaque $^{[8,16,18]}$. Following periodontal therapy, an increase in coccoid bacteria and a decrease in spirochetes and motile rods has been observed ${ }^{[19-21]}$. It has also been suggested that subjects with treated periodontitis, with elevated levels of spirochetes and/or motile rods, have more frequent localized recurrences of periodontitis than patients with low proportions of these morphotypes ${ }^{[16]}$.

A number of antibiotics have been evaluated as aids to periodontal therapy. In particular, extensive studies have been conducted with metranidazole and tetracyclines ${ }^{[7,9-12,22,23]}$. Due to their high concentration in gingival crevicular fluid, their ability to bind to bone and inhibit tissue collagenase, tetracyclines are the most recommended group of antibiotics for treatment of periodontal disease ${ }^{[13,24,25]}$. Among the tetracyclines, doxycycline is a useful derivative, since it is better absorbed than tetracycline and oxytetracycline, and has longer-lasting antibacterial activity in serum, permitting the use of lower doses and less frequent 
administration. It has also been suggested to be the most active tetracycline in vitro against oral bacteria ${ }^{[26]}$.

In the present study, doxycycline was administered systemically to patients with periodontal breakdown, and changes in the subgingival microbial flora were investigated by dark-field microscopy. A significant increase in the percentage of coccoid bacteria and a significant decrease in the percentages of spirochetes, motile rods and other non-motile rods were found after 1 week of drug administration. The present findings are in accordance with previous studies that have shown the same significant effect of tetracyclines on subgingival microbial flora morphotypes ${ }^{[7,9,10,21,23]}$. Since spirochetes and motile rods are considered to have important roles in the pathogenesis of periodontal disease ${ }^{[6,8,16,23]}$, the decrease in the percentages of these microorganisms in the subgingival microbial flora from periodontally diseased pockets would have important implications for periodontal treatment. Therefore, it can be suggested that doxycycline has a signigicant effect on the morphotypes of pathological bacteria associated with periodontal disease, and it can be recommended as an adjunct to traditional periodontal therapy.

Although significant changes were observed in subgingival microbial plaque morphotypes, no apparent changes could be detected in any of the clinical parameters recorded. It is suggested that antibiotics are not indicated for plaque control or treatment of gingivitis. Also their clinical significance for reduction of pocket depth and attachment levels is inconclusive ${ }^{[27]}$. The findings of this study suggest that these clinical parameters were not immediately and directly affected by microbial changes and antibiotic administration. The lack of correlation between clinical parameters and microbiological data may also have been due to the continuing accumulation of supragingival plaque, and also to the limited observation period employed in the present study.

A local and systemic chemotherapeutic approach to the prevention and management of periodontal disease appears to be the trend for the future. This type of therapy has not yet replaced the traditional way of dealing with periodontal disease $^{[11]}$. Although in the present study, doxycycline was found to be active against susceptible microorganisms associated with periodontal disease, it was not suggested to be an alternative to traditional periodontal therapy. However, it could be available as an adjunct to conventional periodontal treatment procedures because of its good absorption, high serum concentration, effective antibacterial activity and low dose requirements.

When recommending doxycycline, the short-term observation period of the present study should also be borne in mind. The present findings do not provide any data on the long-term effects of doxycycline therapy. Therefore, to fully understand the duration of its significant antibacterial effect and the clinical benefit of doxycycline, long-term studies are needed.

\section{Conclusions}

1) Doxycycline administration for 1 week resulted in a significant increase in the percentage of coccoid bacteria.

2) Contrary to the increase of coccoid bacteria, the proportions of spirochetes, 
motile rods and non-motile rods significantly decreased.

3) Although doxycycline had a prominent effect on the morphotypes of subgingival microbial flora, it had almost no effect on clinical parameters.

4) Based on the present findings, doxycycline is recommended as an adjunct to traditional periodontal treatment procedures.

\section{References}

[1] Van Oosten, M. A. C., Hug, H. U., Mikx, F. H. M. and Renggli, H. H.: The effect of amoxicillin on destructive periodontitis. A case report, J. Periodontol., 57, 613-616, 1986

[2] Listgarten, M. A. and Levin, S.: Positive correlation between the proportions of subgingival spirochetes and motile bacteria and susceptibility of human subjects to periodontal deterioration, J. Clin. Periodontol., 8, 122-138, 1981

[3] Socransky, S. S.: Microbiology of periodontal disease-present status and future considerations, J. Periodontol., 48, 497-504, 1977

[4] Slots, J., Mashimo, P., Levine, M. J. and Genco, R. J.: Periodontal therapy in humans. 1microbiological and clinical effects of a single course of periodontal scaling and root planing, and of adjunctive tetracycline therapy, J. Periodontol., 50, 495-509, 1979

[5] Slots, J.: Subgingival microflora and periodontal disease, J. Clin. Periodontol., 6, 351-382, 1979

[6] Armitage, G. J., Dickinson, W. R., Jenderseck, R. S., Levine, S. M. and Chambers, D. W.: Relationship between the percentage of subgingival spirochetes and the severity of periodontal disease, J. Periodontol., 53, 550-556, 1982

[7] Lindhe, J., Liljenberg, B. and Adielsson, E.: Effect of long-term tetracycline therapy on human periodontal disease, J, Clin. Periodontol., 10, 590-601, 1983

[8] Addy, M., Newman, H., Langeroudi, M. and Gho, J. G. L.: Dark-field microscopy of the microflora of plaque, Br. Dent. J., 155, 269-273, 1983

[9] Genco, R. J.: Antibiotics in the treatment of human periodontal diseases, J. Periodontol., 52, 545-558, 1981

[10] Ciancio, S. G., Slots, J., Reynolds, H. S., Zambon, J. J. and McKenna, J. D.: The effect of short-term administration of minocycline $\mathrm{HCl}$ on gingival inflammation and subgingival microflora, J. Periodontol., 53, 557-561, 1982

[11] Moskow, B. S.: Repair of extensive periodontal defect after tetracycline administration, A case report, J. Periodontol., 57, 29-34, 1983

[12] Ciancio, S. G.: Chemotherapeutic agents and periodontal therapy. Their impact on clinical practice, J. Periodontol., 57, 108-111, 1986

[13] Drug Information 88: American Society of Hospital Pharmacists, 318, 320, 327, 328, 1988

[14] Physicians Desk Reference: Barnhart, E. R., 1568, 1987

[15] Löe, H.: The gingival index, the plaque index and the retention index systems, J. Periodontol., 38, 610-616, 1967

[16] Listgarten, M. A. and Hellden, L.: Relative distribution of bacteria of clinically healthy and periodontally diseased sites in humans, J. Clin. Periodontol., 5, 111-132, 1978

[17] Colton, T.: Statistics in Medicine. Little, Brown and Company, Boston, 220, 1974

[18] Listgarten, M. A.: Structure of microbial flora associated with periodontal health and disease in man, J. Periodontol., 47, 1-18, 1976

[19] Philström, B. L., Ortiz-Campos, C. and McHugh, R. B.: A randomized four-year study of periodontal therapy, J. Periodontol., 52, 227-242, 1981

[20] Zappa, V. E., Polson, A. M., Eisenberg, A. D. and Espeland, M. A.: Microbial populations and active tissue destruction in experimental periodontitis, J. Clin. Periodontol., 13, $117-125,1986$

[21] Listgarten, M. A., Lindhe, J. and Hellden, L.: Effect of tetracycline and/or scaling on human periodontal disease. Clinical, microbiological and histopathological observations, J. Clin. Periodontol., 5, 246-271, 1978

[22] Britt, M. R. and Pohlod, D. J.: Serum and crevicular fluid concentrations after a single oral 
dose of metranidazole, J. Periodontol., 57, 104-107, 1986

[23] Silverstein, L., Bissada, N., Pour-Manoucher, M. and Greenwell, H.: Clinical and microbiological effects of local tetracycline irrigation on periodontitis, J. Periodontol., 59, 301-305, 1988

[24] Lindhe, J.: Textbook of Clinical Periodontology. Munskgaard, Copenhagen, Kobenshauns Kliche and Offset., 338, 1983

[25] Golub, L. M., Wollf, M., Lee, H. M., McNamara, T. F., Ramamurthy, T. F., Zambon, J. and Ciancio, S.: Further evidence that tetracyclines inhibit collagenase activity in human crevicular fluid and from other mammalian sources, J. Periodont. Res., 20, 12-23, 1985

[26] Bystedt, H., Dornbush, K. and Nord, C. E.: Concentrations of oxytetracycline, tetracycline and doxycycline in mandibular osteitis, Scand. J. Infect. Dis., Suppl 9, 37-41, 1976

[27] Proceedings of the World Workshop in Clinical Periodontics: Princeton, New Jersey, American Academy of Periodontology, 2-4, 1989 